This is an author produced version of a paper published in International Journal of Nursing Studies. This paper has been peer-reviewed but does not include the final publisher proof-corrections or journal pagination.

Citation for the published paper:

Wann-Hansson, C and Hallberg, I R and Klevsgård, R and Andersson, E "Patients' experiences of living with peripheral arterial disease awaiting intervention: a qualitative study"

Int J Nurs Stud. 2005 Nov;42(8):851-62.

http://dx.doi.org/10.1016/j.ijnurstu.2004.11.009.

Access to the published version may require journal subscription.

Published with permission from: Elsevier 


\title{
Patients' experiences of living with peripheral arterial disease awaiting intervention: a qualitative study
}

\author{
C Wann-Hansson ${ }^{1}$, I R Hallberg ${ }^{1,2}$, R Klevsgård $^{1}$, E Andersson ${ }^{1,2}$
}

Department of Nursing Science, Lund University ${ }^{1}$, The Vårdal Institute, The Swedish Institute for Health Sciences, Lund $^{2}$

Correspondence to: Christine Wann-Hansson, Department of Nursing Science, Lund University P.O. Box 157, SE-221 00 Lund, Sweden

Tel. +46-462221929 Fax: +46-462221935

E mail: christine.wann.hansson@hs.mah.se

Category: Original article 


\begin{abstract}
The aim of this study was to investigate patients' experiences of living with peripheral arterial disease (PAD) and the influence on activities of daily living. Twenty-four patients with various degrees of PAD were interviewed. The transcribed texts were analysed using manifest and latent content analysis. Living with PAD meant carrying a hard-to-bear physical, social and emotional burden, and struggling for relief. The experience of burden was interpreted in the following themes representing consequences and strategies for gaining control in daily life: (I) "being limited by the burden” (II) “striving to relieve the burden” (III) “accepting and adapting to the feeling of burden”. The use of different coping strategies was crucial to achieve some relief. Pain and sleep disturbance emerged as a major feature of living with PAD, and by combining both analgesics and non-pharmacological methods some pain relief was received. To provide optimal alleviation of pain for these patients, education about pain and pain management is of great importance followed by regular evaluations of the pain and pain management. Furthermore, the study underlines the importance of preventing the progression of the vascular disease and from the individual power and knowledge support and preserve as independent life as possible.
\end{abstract}

Keywords: Peripheral arterial disease, Nursing, Content analysis 


\section{Introduction}

Few studies up to now have investigated the experience of living with peripheral arterial disease (PAD) and its impact on daily life. A deeper understanding of having to live with the consequences of PAD is warranted. Several studies in which standardised measures have been used have shown that PAD seriously impairs the quality of life, especially due to problems with pain, reduced energy and restricted mobility (Klevsgård et al., 1999; Chetter et al., 1997; Tretinyak et al., 2000; Breek et al., 2001). Although these results provide an understanding of impaired quality of life and its antecedents it is unclear whether these instruments really reflect all areas of relevance, and they say little about the experience of living with PAD. Further knowledge is needed about the patients' view of living with PAD, which could be helpful in planning and implementing effective, individualised care for them.

PAD is a manifestation of atherosclerosis, and $5 \%$ of men and $2.5 \%$ of women above the age of 60 have symptoms of intermittent claudication (Weitz et al., 1996). It progresses rather slowly and about 5\% need a vascular intervention over a 5-year period after the initial diagnosis (Dormandy and Rutherford, 2000). The symptoms range from intermittent claudication to rest pain, ulceration and gangrene. A majority of the patients are elderly and have a widespread arterial disease with a significantly increased risk of stroke, myocardial infarction and cardiovascular death (Weitz et al., 1996). The most common treatment for patients with intermittent claudication is regular exercise training; a minority of patients deteriorate to a degree where some vascular intervention is considered (Dormandy and Rutherford, 2000). Patients with critical ischaemia, however, always should be considered for active treatment to relieve symptoms, improve functional disability and prevent limb loss (Dormandy and Rutherford, 2000). Despite the development of more effective medical and surgical treatments in the last two decades, PAD is still not possible to cure and vascular treatments are directed towards relief of symptoms and hence improvement in quality of life 
(Powers et al., 1999). This emphasises the importance of understanding areas that are significant for patients with PAD to help them maintain physical function and independence in daily life.

Treat-Jacobson et al. (2002) interviewed 38 patients with PAD to establish a foundation for developing a disease-specific quality of life instrument. Physical, psychosocial and emotional disability and a lack of control over the disease were reported and contributed to a sense of frustration, helplessness and despair (Treat-Jacobson et al., 2002). There was, however, little information about symptoms and how the patients managed to live under these circumstances, which is valuable information for instrument development. It was also unclear whether these patients had undergone vascular treatment or not. Another study, based on interviews with nine patients with PAD 3-18 months after a revascularisation, revealed an experience of pain and powerlessness and that living with PAD puts extensive demands on an individual's power resources (Gibson and Kenrick, 1998). The findings also suggested that the acute medical management of PAD often leads to unrealistic expectations and the patients tended to view their situation in terms of acute illness (Gibson and Kenrick, 1998), even though the experience of living with PAD may be atypical of chronic illness in general. Little is known about how these patients experience the vascular disease course while waiting for treatment. This period is considered, as an acute phase of the chronic disease, and information and support must meet needs related to both the chronic nature and the acute surgical phase of the disease.

Using different coping strategies is the common way for individuals with chronic illness to be and feel in control of what happens to them (Miller, 1992). Coping involves the constantly changing cognitive and behavioural efforts used to manage specific external and/or internal demands that are appraised as taxing and that exceed the resources of the person (Lazarus and Folkman, 1984, p. 141). For a sick person coping refers to handling situations 
that present a threat to the individual, such as dealing with pain, perceived alterations in role function and threat of surgery (Miller, 1992, p. 20). Leech (1982) found that patients with PAD waiting for intervention had difficulties in coping with altered self-image and role function, feelings of uselessness, frustration and depression and a need to have a sense of control over the future. It is therefore important to try to meet the patients' expectations before treatment, with information and support to help them overcome the uncertainty about the outcome and the progression of the disease to obstruct stress and anxiety.

Although a number of studies examined patients with PAD and showed that their quality of life was impaired, knowledge about how they experience their situation and handle problems caused by the disease is still insufficient. Investigations focused on experiences and coping strategies in patients with PAD are needed. The results of any such investigations could give new insights into the requirements for patient-orientated care planning and in the development of methods for this group of patients.

\section{Aim}

The aim of this study was to investigate patients' experiences of living with peripheral arterial disease and the influence on activities in daily life awaiting intervention.

\section{Method}

\subsection{Participants}

A purposive sampling technique was used to recruit 4 patients with severe claudication intermittence and 20 with critical ischaemia who offered different perspectives on the experience of living with PAD. The mean age was 77 years (range 60-92 years) and there were 12 women and 12 men. The severity of patients’ PAD was graded according to 
suggested standards for reporting grade of PAD (Rutherford et al., 1997). Data concerning one or more risk factors were collected from patients’ medical records. Nine of the patients had undergone earlier vascular surgical treatments (Table 1). The inclusion criteria were patients with PAD admitted for planned active treatment at a university hospital in southern Sweden, able to participate in interviews (no serious mental and/or linguistic disorder) and not suffering from other serious disorders that might overshadow the experience of PAD. Fiftytwo potential patients satisfying the inclusion criteria were invited to participate, twelve patients did not want to participate, six patients could not be contacted, nine patients were not able to participate because of too short notice between the summons and the time for admission to hospital and one patient had to be excluded because of a failed tape-recording. The sample size was determined by the rule of redundancy (Morse, 1994) and little new information was obtained in the last five interviews, which confirmed previously collected data.

\subsection{Procedure}

An information letter was distributed by a registered nurse responsible for the planning of admission to the vascular surgical unit. Thereafter oral information was given by the first author by telephone, emphasising the voluntary nature of the study, and for those who agreed to participate a time for interview was decided. Written consent was obtained in conjunction with the interview. The Ethics Committee of the Medical Faculty, Lund University, approved the study (LU 333-01).

All interviews were tape-recorded and performed in the respondent's home by the first author (lasting 45-90 minutes). According to Mishler (1986) interviews should be seen as forms of discourse, constructed jointly by interviewers and respondents, and with the meaning 
of questions and answers contextually grounded. With this as a starting point the respondents were invited to talk openly about their experiences and how the circulation problems in the leg affected their daily life. The opening question was "I am interested in what it is like to live with circulation problems in the legs. Could you please tell me about your experience?” They were also asked to talk about strategies that they used to handle the disease. An interview schedule was used to encircle areas of interest such as pain, sleep, mobility, emotions, energy and social life. To elucidate the meaning of significant experiences, questions such as How did you experience that? What did you think then? How did you react? What did you do and what did you feel? were used. The interviews were transcribed verbatim including non-verbal expressions such as silence and laughter.

\subsection{Analysis}

The texts were analysed using manifest and latent content analysis (Berg, 2001; Cantazaro, 1988). Content analysis has been defined as a research technique that uses a set of procedures to make valid inferences from a text (Weber, 1994). Manifest content analysis illuminates the surface structure in the text and the latent content analysis is extended to an interpretative reading of the symbolism underlying the physical data (Berg, 2001, p. 242). Manifest and latent content analysis includes identification of patterns, which requires that the investigator selects, focuses, simplifies, abstracts and transforms raw data so as to make sense of it (Cantazaro, 1988). Manifest content analysis was used to elicit the perspective of the patients' descriptions, which was physically present in the text. The latent content analysis was used to illuminate the experience of living with PAD and the influence on patients' daily life. Berg (2001, p. 243) suggests that the best solution is to use both manifest and latent content whenever it is possible. 
In the first phase the text was read and reread with efforts to understand each interview as a whole and to grasp words or phrases carrying a meaning of importance for living with PAD (Sandelowski, 1995). The patients talked about consequences of PAD, describing their experience of pain, restricted mobility and problems in performing daily activities. Three authors (C.W.H., R.K. and E.A.) independently performed the first reading of the text. In the next phase the text was divided into meaning units. Each meaning unit was valued from the questions “what is it about?”, “what does it mean?” and "what effect does it have”?. Frequently occurring meaning units that appeared to deal with the same content were organised into sub-themes. The sub-themes were then judged according to how the meaning units in each sub-theme fitted together in a meaningful way and to what extent the differences were clear. Similar sub-themes were brought into themes. To arrive at an interpretation and to identify themes and sub-themes, the statements in each category were analysed critically and questioned, read and compared (Table 2). Finally the three authors (C.W.H., R.K. and E.A.) together decided on a model for analysis which was then presented and discussed with the second author (I.R.H.). The researchers then reflected on and discussed their findings, taking the research question and their preunderstanding into account, and decided on the main theme, themes and sub-themes.

\section{Findings}

Living with peripheral arterial disease meant carrying a hard-to-bear burden and struggling for relief. The experience of burden was interpreted in the following themes representing consequences and strategies for gaining control in daily life: (I) "being limited by the burden” (II) "striving to relieve the burden” (III) “accepting and adapting to the feeling of burden”. 
These themes were related to each other by means of internal variations shown in nine subthemes (Table 3).

\subsection{Being limited by the burden}

The texts were interpreted as if the affected leg was a burden, which meant limitations in physical, social and role functioning and a changed life situation. Mobility became increasingly restricted, which also restricted the ability to participate in social activities. From the narratives of limitations the following sub-themes emerged: being immobile, fatigue and powerlessness, being isolated and being emotionally influenced.

\subsubsection{Being immobile}

The texts revealed experiences involving sensations and perceptions from the leg, such as heaviness, deadness and coldness, and were interpreted as an altered body image which made it difficult to move and the leg did not respond in the usual way. The loss of mobility in the affected limb meant compromised independence and limited daily activities such as gardening, housework, shopping and cleaning, which meant that it became necessary to ask for help with these concerns. Exercise such as cycling and walking had to be slow and for short distances only or completely given up. This also meant difficulties completing physical tasks and participating in social activities. The need for walking assistance such as sticks and walking frames involved a feeling of restriction and were a sign of being old.

Then I have my husband, he is my attendant and he cooks and cleans and generally does everything. (p. 25).

Short distances, yes. Yesterday it was hopeless. I could walk just a few steps then I had to stop and rest for a while, then I could walk a few metres again, so you know your limits. (p. 8). 
Yes I can hardly go to the bathroom now but I still get there. I have a path I use so it still works. You have to fight against the pain. (p. 12).

\subsubsection{Fatigue and powerlessness}

Living with PAD meant a loss of energy with fatigue and powerlessness as a result. Various degrees of sleep disturbances were commonly expressed and the nights were sometimes described as dreadful. Experiences such as being forced to sit in a chair during the nights to stand the pain contributed to tiredness and feelings of exhaustion. The lack of energy meant difficulties performing daily activities, and feelings of being useless were expressed.

Yes, it's just like it has taken the strength and power from me... No, because I never sleep a whole night, I don’t. (p. 19).

I have to force myself to do everything, you have to cook and do some washing and so on. (p. 12).

From being a very active person is it very tough. You have to fight with it and think that you can't be so weak, you have to do this and that but you can't. You have to give up. (p. 18).

\subsubsection{Being isolated}

The texts disclosed limitations in social life and feelings of involuntary isolation. Despite the severity of the ischaemia, the fact of not being able to move outside was experienced as restricting freedom of action. Also, being alone aggravated the sense of isolation and loneliness. Limitations in social activities were described as missing not being able to go to a party or visit friends, to go to the theatre or a restaurant. Loss of interest and inspiration was also a major problem that contributed to the involuntary isolation.

Yes, on excursions and things like that you manage to walk but the next moment everyone else is gone because you can't keep up with the others. It's things like that which play an important part. That you don't have the energy. (p. 13)

No, I've become a home-bird who mostly stays at home. Unfortunately that's the way it is. (p. 25). 


\subsubsection{Being emotionally influenced}

Emotional experiences such as changes in mood and temper and feelings of depression emerged from the texts. The sense that other people often were unaware of the pain and the suffering was one reason for being irritated and it had an impact on relationships with family and friends. The family role changed from being an authority who helped others to becoming the one who needed help, which contributed to a feeling of uselessness and despair. The respondents' lives and thoughts were also marked by uncertainty and fear about the future course and probable outcome of the disease. The fear of amputation was an implicit threat.

And I have been very much afraid that this would cause trouble so that they have to take some of my toes or something like that (p. 7).

Because it influences your temper and everything. Yes everything there is, you lose heart when you are used to being in constant motion. (p. 13).

\subsection{Striving to relieve the burden}

This theme illuminates the respondents' endeavour to relieve the burden and the various strategies invented to handle the disease and the impact on daily life. The strategies were used with patience and inventiveness to gain control over loss of body functions and loss of independence. This meant that a certain extent of relief from the burden was achieved. The following sub-themes emerged from the descriptions of strategies used to relieve the burden: relieving pain and promoting circulation, managing the non-healing wounds, and struggling against the loss of independence.

\subsubsection{Relieving pain and promoting circulation}

Pain was the most commonly occurring symptom, experienced by all respondents. It was found to be unpredictable because it could vary from good to bad days with different degrees of difficulty and had a major impact on daily life. Strategies to relieve the pain and promote 
circulation were interpreted as fostering a sense of control. Vivid descriptions of the pain were given as burning, throbbing, pressure, bursting, stabbing, and a terrible cramp. The pain was also described in metaphors like an electric shock or like thousands of needles or a knife stuck into the foot.

Well, the pain I now have in my foot is something like if you think you turn on a switch so the electricity hits you down there. It's hard to describe. (p. 15).

It feels exactly as if the skin of the toe is too short and when you step on it, oh it is awful and it pricks like needles in the toe. Yes, it's an unpleasant feeling. (p. 4).

Yes, it's just like you can get real pricking sensations which go out into the toes, then it can disappear and then the pain in between, like, it's not that you're cold exactly, but it's like a shooting pain and so on. I don’t know. (p. 20).

Although analgesics were used, a fear of taking pills was commonly expressed. Side effects such as dizziness, indisposition and confusion were common explanations for restrictive use. Instead alternative strategies were devised, for instance dangling the leg over the side of the bed, and the respondents were pleased to have thought of this strategy by themselves. No one has told them about the effect the law of gravity has on peripheral circulation. Different activities such as actively moving the foot or the leg or lying down cycling in the air were tried to promote circulation. The respondents also tried to massage, rub and smear the foot or leg, and touch had an alleviating effect. Distracting activities such as reading, watching television and listening to the radio served to put the pain out of mind for a while.

I am terribly afraid of pills. It sometimes happens during the nights when it hurts that I take a couple of Alvedon, no more. No, I don't like pills. (p. 17).

Yes you have to find your own ideas. I have my whole foot, the right foot I have dangling down so that the blood comes down. (p. 12).

I actually lie down and try to think that the pain will now quit soon and for some reason I think it has worked a few times but when you wake up later on you get the same terrible pain again. (p. 15). 


\subsubsection{Managing the non-healing wounds}

Problems with non-healing wounds were experienced as painful and disgusting. The appearance of the leg was also changed and described as blue, red and glossy. There were active or passive strategies for handling non-healing wounds. Active strategies were applied by those who carefully looked after the wounds and tried different bandages to relieve the pain. The passive strategy was to let the professionals take care of the wounds and attempt not to be involved. It was important to relieve the wound of pressure and to obtain proper and suitable shoes.

It's troublesome because it runs. That's the hard thing and sometimes it hurts. It's hard to wear shoes. (p. 4).

Well, the district nurse came and looked at it and bandaged it. Then it was all right and then it came back, and then it was all right and then it came back again, so it is there now. (p. 25).

\subsubsection{Struggling against the loss of independence}

Being independent of others in handling daily life meant a feeling of control. To avoid and minimise the level of dependency, different strategies were described, such as taking time to rest and modifying routines in daily activities and doing some housework to a reduced extent. Walks and other activities were adapted to capacity and pursued in places where it was possible to rest. Another strategy was not to accept the limitations and struggle to live as normally as possible. Regular training and walking exercises to keep in good shape were another controlling strategy. New solutions, for example, wheels on chairs, sitting instead of standing, were strategies for feeling independent and gaining control.

I don't want to go to places where there is nowhere to sit down. I get this terrible cramp in my legs and then I don't know where to go. So we usually go out to the sea, you know, to Lomma and take walks. And there we walk a long distance and there are certain places where I can sit down and rest when it hurts terribly. Then I sit down for a while and just look out over the sea before we continue our walk. (p. 5).

We have bought it ourselves so it's our chair and it's really good. I can reverse with it, and forwards so that I can wash. My daughter says, "But I can come home and help you.” But I 
say I don't want that. When I can't do it anymore then I'll ask for your help. I think that you feel you have more dignity if you can do things by yourself. I think it would be awful if I couldn't do anything. (p. 10).

\subsection{Accepting and adapting to the feeling of burden}

The experience of living with PAD resulted in a need to understand, adapt and to a certain extent accept the physical influence of the disease. Physical limitations were adapted to over time and the respondents learned by trial and error what the limits were and allowed for dayto-day variations. Social support from spouses, children, friends and other relatives was emphasised in the adaptation process. Limitations in life were perhaps easier to meet as an old person, and age was given as one reason for the feeling of powerlessness, lack of hobbies and decreased social life. Reaching acceptance was interpreted as a more or less deliberately active process followed by reorientation or a passive process with resignation, and from these descriptions the following sub-themes emerged: accepting with reorientation and accepting with resignation.

\subsubsection{Accepting with reorientation}

The texts reflected varying abilities to adapt the lifestyles and continue with life despite the disease. Much of the daily life could be handled by allowing activities to take time and adjusting them to the present state. Social relationships were adjusted by keeping contact by telephone or inviting people home rather than going out. New interests were found that compensated for the loss of other interests that had been crucial before. It was common to think positively; the respondents encouraged themselves not to give up and compared themselves with others whose situation was worse. Adjustments were interpreted as gaining knowledge about abilities that helped the prioritising of possible activities. 
There are many that can't move at all and then. Yes, I always have as a point of reference that there are people who are much worse off than me. And if you are 77 years old and have one year left to normal, what is it called, life expectancy for a Swedish man. (p. 16).

...you had to give up everything and I have became interested in reading instead. I read an awful lot of books. (p. 2).

\subsubsection{Accepting with resignation}

Accepting with resignation was interpreted from the texts as a process including being realistic, facing up to problems and lowering expectations. Loss of interest and a lack of ability to concentrate meant a loss of things that were central before and were not replaced by others. It was accepted that some things belonged to the past and memories from earlier in life helped the respondents to move on and filled them with pleasure. Accepting and resigning was also described as being old and admitting the influence of ageing. The texts revealed a feeling of relief when the diagnosis was established. It had a psychological effect to finally have a time set for hospital admission, as a heavy burden had been lifted from their shoulders. The texts showed feelings of confidence and certitude and an almost unquestioning faith in health care professionals and the interpretation was that the responsibility was surrendered into someone else’s hands.

Now I have had so many experiences in my life so if it is a year or something like that when I can't, then I'll take that. You have to accept it. (p. 16)

Before there was the uncertainty and not knowing if you'd get any help or not. It was hopeless but now I know that tomorrow I have to go there, on Thursday I have to go there. They are taking care of me, you don't have to think about it anymore. (p. 18)

\section{Discussion}

This finding suggests that peripheral arterial disease has a major influence on daily life and can be understood as carrying a hard to bear burden and a struggling for relief. Being limited by the burden was illuminated through the sub-themes being immobile, fatigue and 
powerlessness, being isolated and being emotionally influenced. Various strategies were invented in the striving to relieve the burden which meant relieving pain and promoting circulation, managing the non-healing wounds and struggling against the loss of independence. Accepting and adapting to the feeling of burden was a more or less deliberate active process and was illuminated through the sub-themes accepting with reorientation and accepting with resignation.

\subsection{Methodological considerations}

In qualitative research the findings should be evaluated in terms of trustworthiness, which replaces conventional criteria such as internal and external validity, reliability and objectivity (Polit and Hungler, 1999). Trustworthiness deals with credibility, transferability, dependability and confirmability (Lincoln and Guba, 1985). To increase the credibility, all interviews were conducted in the respondent's home to make them feel safe and secure. A sufficient amount of time was allotted for the interviewer to understand the context of the respondents and to create opportunities for the respondents and investigator to get to know each other and thereby increase the respondents' feeling of confidence. A balance of power in the interview situation is important to elicit more valid descriptions of individual experiences (Mishler, 1986).

The number of respondents is likely to be considered sufficient since more than one informant provided the same information or verified responses from one another. A majority of the respondent were over 75 years old, which could imply that their experiences are affected by other conditions as well. However, age is one of the most common risk factors for PAD and it is well known that this group of patients often have other health problems caused 
by the arteriosclerotic disease (Dormandy and Rutherford, 2000) and is therefore not a reason for eliminating these patients from studies of this kind.

The first author strove to become immersed in the data during the analysis process by reading and rereading the texts and continuously returning to the text during the categorising, while also consulting the other authors. Multiple meanings are always present in data and there is no right meaning, only the most accurate meaning from a particular perspective (Downe-Wamboldt, 1992). There is a risk that the researchers' preunderstanding influenced the analysis. The preunderstanding of the content in the text of the first author was her experiences as a nurse at a vascular surgical unit, however, the encounter with the respondents was in the capacity as a researcher. In an attempt to limit this risk of predetermined interpretation and to increase the credibility, a consistent method throughout the research process was used with the analysis focused on the text and using other researchers in the process of analysis (Cantazaro, 1988; Downe-Wamboldt, 1992). Further, dependability in the analysis of the findings was confirmed by the fact that the last author (EA) had not participated in the study prior to this point. All she had to rely on was the text of the interviews. To further strengthen the trustworthiness and make the findings open to confirmation, quotations were used to reflect the interpretation of texts.

The transferability of the findings in qualitative studies depends on the degree of similarity between the study population and the actual setting and thus findings cannot be wholly applied to another context (Lincoln and Guba, 1985). In this study the transferability is tied to the fact that all respondents represent the same point of time in their disease, that is, having symptoms from PAD prior to a vascular treatment which made them focused on the research question. The respondents represented both men and women with a range of age from 60-92 and various degrees of PAD, which meant that persons with more severe PAD were also 
included. This suggests that the findings of this study can most likely be transferred to other persons with PAD at resembling age and severity of PAD prior to treatment.

\subsection{Discussion of the findings}

Living with PAD meant social limitations and emotional experiences, which contributed to the sense of burden. Involuntary isolation and uncertainty and fear about the future and possible outcomes influenced the life situation and meant a lack of control over the disease progression. Other studies have shown similar results from interviews with patients with PAD, i.e. feelings of isolation and fear of loss of function and independence (Hallin et al., 2002; Treat-Jacobson et al., 2002). However, extensive effect on social life and emotional impact has not commonly been documented in the literature. On the contrary, several studies have not been able to demonstrate any significant difference in social isolation or mental health between patients with PAD and controls by using different generic quality of life instruments (Khaira et al., 1996; Breek et al., 2001; Taft et al., 2001). Generic quality of life instruments have a broad application in healthy as well as ill populations and reflect different aspects of emotional and social activities, which are not directly related to health. This could be one reason why these instruments do not have the sensitivity to detect the impact on social life and the emotional impact in patients with PAD. Using generic quality of life instruments thus entails a potential risk that these aspects may be neglected and considered not to be a significant problem for this group of patients. The findings of this study further confirm the necessity of acquiring an overall picture of the experience of living with PAD through comprehensive individualised assessment. From a clinical perspective it seems important also to take the social and emotional aspects into account to identify specific vulnerable patients and to apply interventions accordingly. 
To choose an active or passive approach to accepting and adapting to the feeling of burden was a more or less deliberate action and stood out as important. These findings could be seen in the light of coping theory as described by Lazarus and Folkman (1984, p. 141), referring to two overriding functions: problem-focused coping when managing or altering problems with the environment which cause distress, and emotion-focused coping when regulating the emotional response to the problem. Problem- and emotion-focused coping influence each other throughout a stressful encounter and can facilitate and impede each other (Lazarus and Folkman, 1984, p. 141). Both problem- and emotion-focused coping were seen in this study either as accepting with reorientation when patients tried to solve problems in their environment and encouraged themselves not to give up, or as accepting with resignation when the natural course of ageing was seen as a contributory factor in limitations in daily life. Another way to accept and adapt to the burden of the PAD was to compare with others whose situation was worse. This form of emotion-focused coping could be explained by "downward comparison”, which is a cognitive process used to enhance subjective well-being by comparison with others whose condition is worse (Wills, 1981). This process has previously been described in a study of adjustment to breast cancer (Wood et al., 1985). Downward comparisons seemed to be most important early in the adjustment process, intended to prevent the victim from being overwhelmed by the frightening new circumstances (Wood et al., 1985). Another study of older people with cancer showed that downward comparisons were made mostly with persons in a worse state with regard to the effects and the old age itself, as a way to endure the cancer (Thomé et al., 2003). In the current study downward comparisons were used to give a more positive view of the changed life situation and helped the patients to accept the limitations with a reorientation to continue with life. This may help patients to protect themselves and reduce the impact of PAD. The findings indicate that the impact of PAD is a source of undesirable stress, which calls for different ways of accepting and 
adapting to the new situation and recapturing control in daily life. This emphasises the importance of being sensitive to individual needs in the support of suitable coping strategies to relieve the burden. This support should include strategies to maintain a sense of normality and keeping signs and symptoms of illness under control, with information about behavioural changes such as smoking cessation, nutrition and exercise in structured education programmes.

Pain stood out as the most influential factor in the respondents' everyday life and was a cause of sleep disturbance among many respondents. Everything revolved around strategies to relieve the pain and promote circulation in an attempt to reduce the impact of pain on daily life and sleep. It has been found that pain is the most frequently experienced symptom in patients with PAD and that suffering from pain contributes to many problems in daily life (Treat-Jacobson et al., 2002; Gibson and Kenrick, 1998). However, further information regarding the nature of the pain and how these patients actually handle their pain is sparse and has to our knowledge not been reported earlier. In this study the patients contributed important knowledge about the ischaemic pain and how by combining medication, distraction and alterations in activity they succeeded in relieving the pain. To have the patients' experience of pain and knowledge of helpful strategies as a starting-point is significant when suitable pain relief methods are planned and applied. This knowledge is valuable when teaching patients about pain and pain management to provide optimal alleviation of the pain, which is one of the most important goals of nursing care in this group of patients.

Apparently a variety of both pharmacological and non-pharmacological methods are required to manage the pain of PAD. It was striking that many of the respondents mentioned a fear of taking analgesics because of side effects or because they thought it was not good for them to take so many pills. This may mean that the patients had more pain than they actually needed to have. Similar findings in older people’s decision making about the management of 
musculoskeletal pain showed that they focused on the negative dimensions of medication and preferred to use a combination of strategies to relieve the pain (Ross et al., 2001). The drug distribution is usually different in older patients because of blood flow to organs, protein binding and body composition that occurs with ageing (Gloth, 2001). This could explain the experiences of side effects described in this study, since the majority of the respondents were over 75 years old. This emphasises the importance of following up the use of prescribed analgesics by pain assessment and noting the compliance and when necessary make dosing adjustments. Here nurses are in a unique position to perform these follow-ups, to monitor, teach and support the patients in the process of finding the optimal drug regimen to maximise effects and minimise side effects in combination with non-pharmacological methods which are considered useful for the individual patient.

Suffering from PAD means living with a threat of dependency and constraints on autonomy in daily life. Striving for independence was important to maintain integrity and dignity. Gibson and Kenrick (1998) also showed the importance of maintaining independence in making everyday decisions and that the participants felt their control was threatened by the PAD. However, earlier studies have not described the way patients handled the dependency and the lack of control. Examples of strategies that were used in this study were changing demands and exerting themselves to do as much as possible and try to create some sort of normality in life. The findings of this study confirm the need for health care professionals to empower the patients to preserve their independence by reducing the progression of the vascular disease. Knowledge and insight about what is happening when suffering from vascular disease enables patients and their families to feel more in control and alleviate anxiety and uncertainty. Thus the main role of health cares in both inpatient and outpatient settings may be to implement interventions to maintain, regain or improve function and prevent disability. 


\section{Conclusion}

Living with peripheral arterial disease means a major impact on daily life and was experienced as a physical, social and emotional burden. Information from the patients' own perspective results in a deeper understanding for this group of patients, and it is obvious that existing questionnaires have not been designed to assess some of the important consequences such as the social and emotional impact of PAD. The use of different coping strategies was crucial to achieve some extent of relief, and the power to choose active or more passive strategies was important for accepting the new life situation. Pain emerged as a major feature of living with PAD, and by combining both analgesics and non-pharmacological methods some pain relief was received. To provide optimal alleviation of the pain for these patients, education about pain and pain management is of great importance followed by regular evaluations of the pain and pain management. Furthermore, the study underlines the importance of preventing the progression of the vascular disease and from the individual capacity and knowledge support and preserve as independent life as possible. This highlights that health promotion to target the specific needs of patients with PAD is warranted. Further research focused on developing and implementing structured information and educational programmes is needed. Lastly, the findings of this study could inform health care professionals to enhance the patient's ability to identify and support the effective coping strategy the patient selects.

\section{Acknowledgements}


This study was supported by the Lori Lindahls Foundation and the Department of Nursing

Science, Lund University, the Vårdal Institute, the Swedish Institute for Health Sciences,

Lund and the Department of Vascular Disease, Malmö University Hospital.

\section{References}

Berg, B.L., 2001. Qualitative research methods for the Social Science, 4th Edition. Allyn and Bacon, Boston, pp. 238-267.

Breek, J.C., Hamming, J.F., De Vries, J., Aquarius, A.E.A.M., van Berge Henegouwen, D.P., 2001. Quality of life in patients with intermittent claudication using the World Health Organisation (WHO) Questionnaire. European Journal of Vascular and Endovascular Surgery 21, 118-122.

Catanzaro, M., 1988. Using qualitative analytical techniques. In: Woods, N.F., Catanzaro, M. (Eds.), Nursing Research. Theory and Practice. The C.V. Mosby Company, St. Louis, pp. 437-456.

Chetter, I.C., Spark, J.I., Dolan, P., Scott, D.J.A., Kester, R.C., 1997. Quality of life analysis in patients with lower limb ischaemia: Suggestions for European standardisation. European Journal of Vascular and Endovascular Surgery 13, 597-604.

Dormandy, J.A., Rutherford, R.B., 2000. Management of Peripheral Arterial Disease (PAD). TransAtlantic Inter-Society Consensus (TASC). European Journal of Vascular and Endovascular Surgery 19 (suppl A), 1-244.

Downe-Wamboldt, B,. 1992. Content analysis: method, applications and issues. Health Care for Women International 13, 313-321.

Gibson, J.M.E., Kenrick, M., 1998. Pain and powerlessness: the experience of living with peripheral vascular disease. Journal of Advanced Nursing 27, 737-745.

Gloth, M.F., 2001. Pain management in older adults: prevention and treatment. Journal of American Geriatrics Society 49, 188-199.

Hallin, A., Bergqvist, D., Fugl-Meyer, K., Holmberg, L., 2002. Areas of Concern, Quality of Life and Life Satisfaction in Patients with Peripheral Vascular Disease. European Journal of Vascular and Endovascular Surgery 24, 255-263.

Khaira, H.S., Hanger, R., Shearman, C.P., 1996. Quality of Life in Patients with Intermittent Claudication. European Journal of Vascular and Endovascular Surgery 11, 65-69.

Klevsgård, R., Hallberg, I.R., Risberg, B., Thomsen, M.B., 1999. Quality of life associated with varying degrees of chronic lower limb ischaemia; Comparison with a healthy sample. European Journal of Vascular and Endovascular Surgery 17, 319-325. 
Lazarus, R.S., Folkman, S,. 1984. Stress, appraisal, and coping. Springer Publishing Company, New York. pp. 141-180.

Leech, J.E., 1982. Psychosocial and physiologic needs of patients with arterial occlusive disease during the preoperative phase of hospitalization. Heart \& Lung 11 (5), 442-449.

Lincoln, Y.S., Guba, E.G., 1985. Naturalistic inquiry. Sage Publications Inc, Newbury Park, California.

Miller, J.F., 1992. Coping with chronic illness. F.A. Davis Company, Philadelphia. pp. 19-49.

Mishler, E.G., 1986. Research Interviewing: Context and Narrative. Harvard University Press, London.

Morse, J.M., 1994. Designing funded qualitative research. In Denzin N.K., Lincoln Y.S. (Eds) Handbook of qualitative research. Sage Publications, Thousand Oaks, California. pp 220235.

Polit, D.F., Hungler, B.P., 1999. Nursing research, Principles and Methods, 6th edition. J.B. Lippincott Company, Philadelphia, PA.

Powers, K.B., Vacek, J.L., Lee, S., 1999. Noninvasive approaches to peripheral vascular disease. Postgraduate Medicine 106 (3), 52-64.

Ross, M.M., Carswell, A., Hing, M., Hollingworth, G., Dalziel, W.B., 2001. Seniors’ decision making about pain management. Journal of Advanced Nursing 35 (3), 442-451.

Rutherford, R.B., Baker, J.D., Ernst, C., Johnston, K.W., Porter, J.M., Ahn. S., et al. 1997. Recommended standards for reports dealing with lower extremity ischemia: Revised version. Journal of Vascular Surgery 26, 517-538.

Sandelovski, M., 1995. Focus on qualitative methods. Qualitative analysis: What it is and how to begin. Research in Nursing \& Health. 18, 371-375.

Taft, C., Karlsson, J., Gelin, J., Jivegård, L., Sandström, R., Arfvidsson, B., Dahllöf, A.G., Lundholm, K., Sullivan, M., 2001. Treatment efficacy of intermittent claudication by invasive therapy, supervised physical exercise training compared to no treatment in unselected randomised patients II: One-year results of health-related quality of life. European Journal of Vascular and Endovascular Surgery. 22, 114-123.

Thomé, B., Dykes, A.K., Gunnars, B., Hallberg, I.R., 2003. The experiences of older people living with cancer. Cancer Nursing 26, 85-96.

Treat-Jacobson, D., Halverson, S.L., Ratchford, A., Regensteiner, J.G., Lindquist, R., Hirsch, A.T., 2002. A patient-derived perspective of health-related quality of life with peripheral arterial disease. Journal of Nursing Scholarship 34, 55-60.

Tretinyak, A.S., Lee, E.S., Kuskowski, M.A., Caldwell, M.P., Santilli, S.M., 2001.

Revascularization and quality of life for patients with limb-threatening ischemia. Annals of Vascular Surgery 15 (1), 84-88. 
Weber, R., 1994. Basic content analysis. Research Practice. In: Lewis-Beck, M. (Ed), International Handbook of Qualitative Applications in the Social Services. Sage 6, London.

Weitz J.I., Byrne J., Clagett G.P., Farkouh M.E., Porter J.M., Sackett D.L., Strandness D.E. \& Taylor L.M. (1996) Diagnosis and treatment of chronic arterial insufficiency of the lower extremities: A critical review. Circulation 94 3026-3049

Wills, T., 1981. Downward comparison principles in social psychology. Psychol Bull 90, 245271.

Wood, J.V., Taylor, S.E., Lichtman, R.R., 1985. Social comparison in adjustment to breast cancer. Journal of Personality and Social Psychology 49, 1169-1183. 
Table 1. Demographic characteristics of the patients $(\mathrm{N}=24)$

Age m (SD)

Sex n (\%)

Male/female

Cohabitation n (\%)

Living alone

Living with relative

Severity of disease $n(\%)$

Intermittent claudication

Ischaemia rest pain

Ischaemia ulcers

$77(7.2)$

$12 / 12(50 / 50)$

7 (30)

$17(70)$

4 (17)

2 (8)

$18(75)$

Risk factors n (\%)

Smoking

Hypertension

$19(79)$

Heart disease

5 (21)

7 (29)

4 (17)

4 (17)

Kidney disease

Previous vascular

interventions $\mathbf{n}(\%)$

$<12$ months ago

$5(21)$

$>12$ months ago

$4(16)$ 
Table 2. The analysis process of interpretation and examples of interpreted meaning units, sub-themes

\begin{tabular}{l}
\hline Meaning units \\
\hline “I can't use the stairs and I can't \\
really do anything here at home” (p. \\
21 ) \\
“No, I could never have managed \\
on my own. It would never have \\
worked. You need to shop almost \\
every day and wash and \\
everything.” (p. 18) \\
"I am afraid to go to bed because it \\
hurts so it happens that I switch on \\
the TV, yes I do. But sometimes I \\
don't put on the TV and just fall \\
asleep here in the chair with that \\
blanket on my knees.” (p. 6) \\
"I have not been anywhere. I \\
haven't wanted to go anywhere \\
really. Well, to my children and the \\
like. Yes, now there was someone \\
who turned 75 but if I have to sit so \\
long I'll have to get up and move \\
(p. 2)
\end{tabular}

"Yes, I have read in the paper that they take off the leg and then I get completely dejected”. (p. 19)

“...then I have to get up and walk around in the apartment. Sometimes I lie down on the floor and kick with my legs. It's also a rather good way. You know, you lie down and cycle in the air and then krrrsch it is like something is starting.” (p. 5)

"I have taken care of it by myself and it has worked out well. I buy Alsol solution at the pharmacy and it works very well. And I keep it clean from bacteria and so. I put some of that on before I put a shoe on. I handle it. I want it to disappear completely.” (p. 4)

"So I think there are many who are worse off than me. It's nothing, I don’t complain.” (p. 2)

“I just think it's wonderful that I have travelled all I could do. It has to end sometime.” (p. 1)

What is it
about?

Restricted

mobility as a consequence of the disease. Compromised independence due to restricted mobility.

Sleep disturbance due to the pain at night.

What does it
mean/What effect
does it have?

Perceived

limitations in
physical function.

$\begin{array}{ll}\text { Perceived } & \text { Being } \\ \text { limitations in daily } & \text { immobile } \\ \text { activities. } & \end{array}$

\section{Limitations in} social life due to the lower limb ischaemia.

Fear about the future and probable outcome.

An alternative strategy to relieve the pain and promote circulation.

A strategy to handle the nonhealing wounds.

Downward comparisons.

Looking back on a life span.
Tiredness and exhaustion in relation to sleep disturbance.

Feeling limited in social life.

Feeling mentally affected by the disease.

Feeling relieved of the pain.

Taking an active part in the treatment.
Fatigue and powerlessness Themes Sub-themes

Being
immobile Being limited by the burden 
MAIN THEME: Carrying a burden that is hard to bear, and struggling for relief.

THEME 1: Being limited by the burden

Sub-themes

- Being immobile

- Fatigue and powerlessness

- Being isolated

- Being emotionally influenced

THEME 2: Striving to relieve the burden

Sub-themes

- Relieving pain and promoting circulation

- Managing the non-healing wounds

- Struggling against the loss of independence

THEME 3: Accepting and adapting to the feeling of burden

Sub-themes

- Accepting with reorientation

- Accepting with resignation 